\section{Point target detection of infrared images with eigentargets}

\author{
Ruiming Liu, ${ }^{a}$ Erqi Liu, ${ }^{b}$ Jie Yang, ${ }^{a}$ Tianhao Zhang, ${ }^{a}$ \\ and Yuan $\mathrm{Cao}^{\mathrm{a}}$ \\ ${ }^{a}$ Shanghai Jiaotong University, Institute of Image \\ Processing and Pattern Recognition, No. 800 Dongchuan \\ Road, Shanghai, 200240, China \\ ${ }^{\mathrm{b}}$ China Aerospace Science and Industry Corporation, \\ Institute of the Second Academy, Beijing, 100854, China \\ E-mail: liuruiming@sjtu.edu.cn
}

\begin{abstract}
A novel detection method for infrared (IR) point targets based on eigentargets is presented. The conception of eigentargets is proposed by making reference to eigenfaces in the field of face recognition. After creating the target training images using the Gaussian intensity function, we refine our method and obtain eigentargets that are a group of eigenvectors of the training set. These eigenvectors are then used to calculate the target map, defined by a target map function, at every location in the image to be detected. The large values in the target map image indicate the presence of targets. In comparison with other commonly used detection methods, our method has better performance with better receiver operating characteristics, and larger signalto-noise ratio (SNR) and background suppression factor. (c) 2007 Society of Photo-Optical Instrumentation Engineers.
\end{abstract} [DOI: 10.1117/1.2802301]

Subject terms: target detection; infrared (IR) images; Gaussian intensity function; principal component analysis (PCA).

Paper 070509LR received Jun. 20, 2007; revised manuscript received Aug. 12, 2007; accepted for publication Aug. 24, 2007; published online Nov. 16, 2007.

\section{Introduction}

For infrared (IR) point targets, the background is usually contaminated by unknown noise, and the target-tobackground contrast is very low, so it is very difficult to detect them. The issue of detecting targets can be viewed as a two-class pattern recognition problem. ${ }^{1}$ An image to be detected consists of a target class and a background class. We can use the pattern recognition algorithms to detect targets. In Ref. 1, the authors detected targets with the Fukunaga Koontz transform (FKT), which is a two-pattern recognition algorithm based on principal component analysis (PCA). Moreover, Chan et al. ${ }^{2}$ use neural networks to detect targets. Turk and Pentland applied PCA to face recognition and detection. ${ }^{3}$ In this letter, we make reference to this method to detect IR point targets.

\section{Eigentargets for Detection}

Consider a set of $N$ training target images $\left\{x_{1}, x_{2}, \ldots, x_{N}\right\}$ that are $m \times m$ in size. We convert them to column vectors $\left\{\Gamma_{1}, \Gamma_{2}, \ldots, \Gamma_{N}\right\}$. The average target is defined by $\psi$ $=(1 / N) \sum_{n=1}^{N} \Gamma_{n}$. Then the covariance matrix can be calculated by

0091-3286/2007/\$25.00 @ 2007 SPIE
$C=\frac{1}{N} \sum_{n=1}^{N}\left(\Gamma_{n}-\psi\right)\left(\Gamma_{n}-\psi\right)^{T}$.

$C$ is factorized into the form

$C=\Phi \Delta \Phi^{T}$,

where $\Phi$ is a matrix with the eigenvectors of $C$, and $\Delta$ is a diagonal matrix with the corresponding eigenvalues as elements of its main diagonal. We assume that the eigenvectors in $\Phi$ have been sorted into $\Phi=\left\{\phi_{1}, \phi_{2}, \ldots, \phi_{N}\right\}$ by descending order of corresponding eigenvalues $\Delta$ $=\left\{\Delta_{1}, \Delta_{2}, \ldots, \Delta_{N}\right\}$. Then the first $M$ eigenvectors

$\Phi_{t}=\left\{\phi_{1}, \phi_{2}, \ldots, \phi_{M}\right\}$,

in $\Phi$ are used to construct a subspace (the target space). We call $\Phi_{t}$ eigentargets. Eigentargets $\Phi_{t}$ represent the main feature of targets.

Given an image to be detected, at every pixel location $(x, y)$, an image vector $\Gamma(x, y)$ is constructed whose size is equal to the size of the training images. This vector is projected into the target space by the following operations:

$\omega_{k}=\phi_{k}^{T}(\Gamma-\psi)$.

$\Omega=\left[\omega_{1}, \omega_{2}, \ldots, \omega_{M}\right]$ describes the contribution of each eigentarget in representing the input target image $\Gamma(x, y)$. Then we can obtain the reconstructed target image by

$\Phi_{r t}=\sum_{k=1}^{M} \omega_{k} \phi_{k}$.

Ordinarily, the Euclidian distance $\epsilon(x, y)$ between the unknown image vector $\Gamma(x, y)$ and the reconstructed target image is used to detect faces in Ref. 3. It is formulated by

$\epsilon(x, y)=\left\|\Gamma-\psi-\Phi_{r t}\right\|<\theta$,

where $\theta$ is a chosen threshold. The value of $\epsilon(x, y)$ is a face map in Ref. 3. The small values indicate the presence of faces. In this letter, we design a new map function to complete our detection task. It is defined by

$\varsigma(x, y)=\exp \left[\epsilon(x, y)^{2} / 2 \sigma^{2}\right]$.

The preceding map, the target map, can let all component values of $s(x, y)$ be elements of $[0,1]$ for allowing meaningful comparisons across widely varying conditions. Moreover, Eq. (7) makes the large values indicate the presence of targets. This is more consistent with our intuition than Eq. (6), whose small values indicate the presence.

Apparently, eigenfaces and our eigentargets methods are supervised learning methods. For all supervised learning methods, the selection of the training set is very important for their performance. There are two approaches to generate the target training images. One is cropping from the real IR images to be detected. The other is using the synthetic images generated by the Gaussian intensity function. ${ }^{4}$ Two reasons support our using synthetic target images to construct the training set. The first reason is that cropping the real target images is difficult and troublesome because we do not know the location of targets before detection. The 


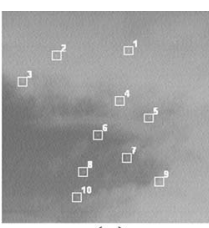

$\left(a_{1}\right)$

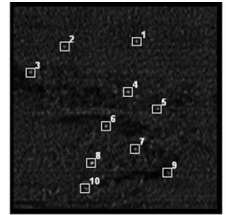

$\left(a_{2}\right)$

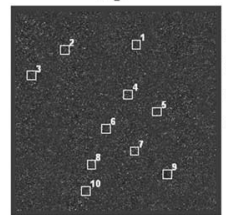

$\left(a_{4}\right)$

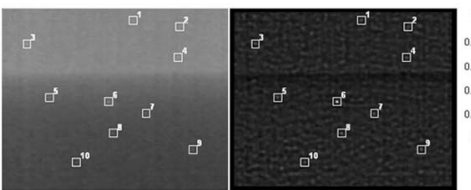

$\left(b_{2}\right)$

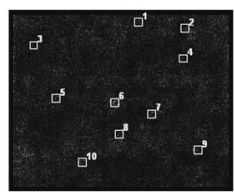

$\left(\mathrm{b}_{4}\right)$

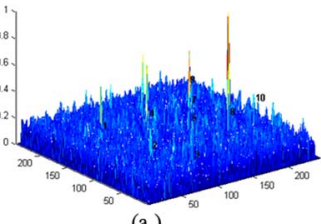

$\left(a_{3}\right)$

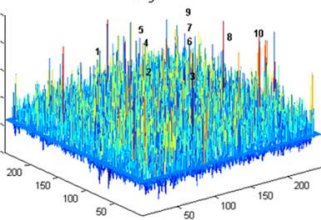

$\left(\mathrm{a}_{5}\right)$

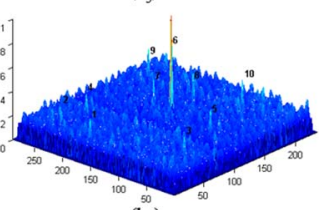

$\left(\mathrm{b}_{3}\right)$

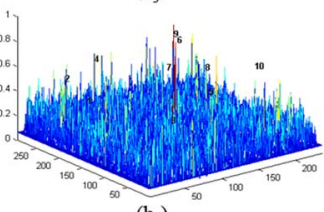

$\left(b_{5}\right)$
Fig. 1 The two experimental IR images and the comparison of the target map. $\left(a_{1}\right)$ and $\left(b_{1}\right)$ are the IR images to be detected. $\left(a_{2}\right)$ and $\left(b_{2}\right)$ are the target maps of eigentargets. $\left(a_{3}\right)$ and $\left(b_{3}\right)$ are their plots, respectively. $\left(a_{4}\right)$ and $\left(b_{4}\right)$ are the target maps of FKT. $\left(a_{5}\right)$ and $\left(b_{5}\right)$ are their plots, respectively.

second reason is that the real images generally are contaminated by noise. Thus, they cannot precisely and correctly represent the feature of targets. The form of the Gaussian intensity function is

$I(x, y)=I_{\max } \exp \left\{\left(-\frac{1}{2}\left[\frac{\left(x-x_{0}\right)^{2}}{\sigma_{x}^{2}}+\frac{\left(y-y_{0}\right)^{2}}{\sigma_{y}^{2}}\right]\right)\right\}$.

The pixel spread characteristics of simulated point targets are defined by the maximum intensity $I_{\max }$, horizontal spread parameter $\sigma_{x}$, and vertical spread parameter $\sigma_{y}$. $\left(x_{0}, y_{0}\right)$ is the center location of the target, and its intensity value is $I_{\max }(x, y)$ is the location of other pixels. In Ref. 4
(Fig. 3), some simulated point targets are shown.

\section{Experiment and Results}

We first compare the detection performance of the Fukunaga Koontz transform (FKT) ${ }^{1,5}$ with that of eigentargets proposed by us under the same experimental conditions. Figure 1 shows the two experimental images to be used in this letter. The targets in Fig. 1 are very dim, so it is difficult to detect them from the background.

Generally speaking, the size of IR point targets is less than 100 pixels, so the size of training images is set as 11 $\times 11$ in our experiments. For detecting potential targets from Fig. 1 $\left(\mathrm{a}_{1}\right)$ and Fig. 1( $\left.\mathrm{b}_{1}\right), 100$ target training images are created randomly by the Gaussian intensity function [Eq. (8)]. The number of eigentargets in Eq. (3) is $M=10$. The parameter in Eq. (7) is experientially set as $\sigma=0.25$. FKT detection is executed according to the contents of Refs. 1 and 5.

From Figs. 1( $\left.a_{4}\right), 1\left(a_{5}\right), 1\left(b_{4}\right)$, and $1\left(b_{5}\right)$, we can see that FKT has some large response points in the background because of the existence of the clutter. That means that its capability of suppressing the background is not strong. Moreover, the peaks at target locations are not distinct. The main cause of these disadvantages is that FKT cannot precisely capture the features of targets and backgrounds so that the shift-version targets and backgrounds also have strong responses, while we expect detection methods have a large response only at the pixel located at the center of the target. Figures $1\left(a_{2}\right), 1\left(a_{3}\right), 1\left(b_{2}\right)$, and $1\left(b_{3}\right)$ show the results of eigentargets proposed by us. We can see that the outputs corresponding to the background pixels are small. That means that eigentargets have a better capability of suppressing the background. Furthermore, eigentargets make distinct peaks at the center location of every target. Thus, we can easily and precisely find the correct locations of targets.

The receiver operating characteristic $(\mathrm{ROC})^{1}$ curve is a good tool for evaluating the performance of a detection method. Figure 2 shows ROC curves of eigentargets and FKT for detecting targets from two images of Fig. 1. The ROC curves are constructed by varying the detection threshold (ROC operating point). In Figs. 2(a) and 2(b), when the false alarm is close to $0 \%$, the lowest detection rate of eigentargets is $80 \%$, while that of FKT is $0 \%$. More-

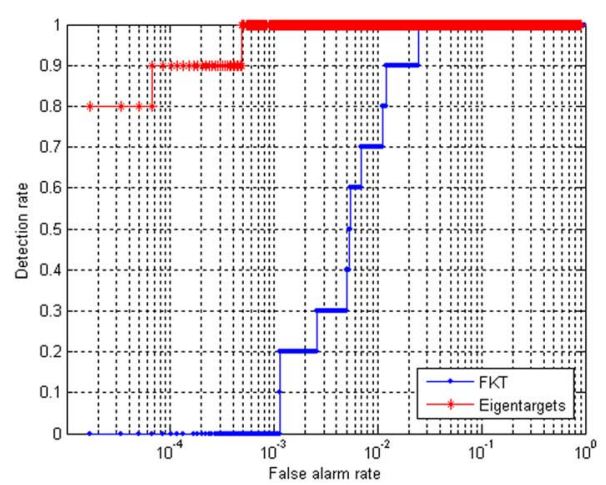

(a)

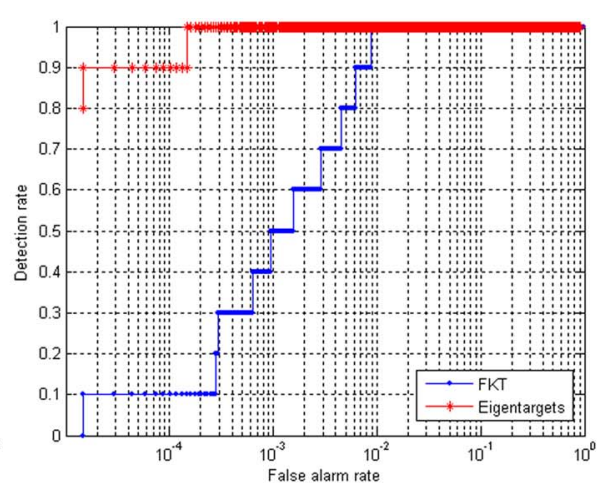

(b)

Fig. 2 The ROC curves of eigentargets and FKT. (a) The ROC curve of detecting Fig. 1(a $\left.a_{1}\right)$. (b) The ROC curve of detecting Fig. $1\left(b_{1}\right)$. 
Table 1 The performance comparison of the different detection methods for detecting targets from the two images of Fig. 1.

\begin{tabular}{|c|c|c|c|c|c|c|c|c|c|c|c|c|c|c|c|c|}
\hline & \multirow[b]{2}{*}{ Target } & \multicolumn{5}{|c|}{$S N R$} & \multicolumn{5}{|c|}{$S N R G$} & \multicolumn{5}{|c|}{$B S F$} \\
\hline & & MMed & MMean & TDLMS & FKT & ET & MMed & MMean & TDLMS & FKT & ET & MMed & MMean & TDLMS & FKT & ET \\
\hline \multirow{10}{*}{$\begin{array}{c}\text { Figure } \\
1\left(a_{1}\right)\end{array}$} & 1 & 0.1271 & 0.1039 & 0.8373 & 0.5915 & 1.4655 & 0.1330 & 0.1087 & 0.8763 & 0.6191 & 1.5338 & \multirow{10}{*}{1.6938} & \multirow{10}{*}{1.7023} & \multirow{10}{*}{0.6443} & \multirow{10}{*}{1.6099} & \multirow{10}{*}{$\underline{2.3257}$} \\
\hline & 2 & 0.1227 & 0.0999 & 0.8167 & 0.5010 & $\underline{1.0260}$ & 0.1370 & 0.1115 & 0.9115 & 0.5412 & $\underline{1.1451}$ & & & & & \\
\hline & 3 & 0.1349 & 0.1143 & 0.8038 & 0.4158 & 1.3644 & 0.1514 & 0.1283 & 0.9020 & 0.4516 & $\underline{1.5311}$ & & & & & \\
\hline & 4 & 0.1365 & 0.1169 & 0.7925 & 0.6004 & $\underline{1.7335}$ & 0.1475 & 0.1263 & 0.8561 & 0.6486 & 1.8726 & & & & & \\
\hline & 5 & 0.1258 & 0.1109 & 0.7724 & 0.4422 & 1.6793 & 0.1494 & 0.1318 & 0.9178 & 0.5018 & $\underline{1.9954}$ & & & & & \\
\hline & 6 & 0.1580 & 0.1446 & 0.6794 & 0.4631 & $\underline{1.9860}$ & 0.2231 & 0.2043 & 0.9598 & 0.5669 & $\underline{2.8055}$ & & & & & \\
\hline & 7 & 0.1575 & 0.1345 & 0.6981 & 0.7119 & $\underline{1.4017}$ & 0.2040 & 0.1742 & 0.9040 & 0.8663 & $\underline{1.8150}$ & & & & & \\
\hline & 8 & 0.1666 & 0.1440 & 0.7069 & 0.9924 & $\underline{2.6705}$ & 0.2229 & 0.1927 & 0.9457 & 1.3276 & $\underline{3.5725}$ & & & & & \\
\hline & 9 & 0.1157 & 0.0998 & 0.8375 & 0.4634 & 0.8821 & 0.1284 & 0.1108 & 0.9295 & 0.4927 & 0.9681 & & & & & \\
\hline & 10 & 0.1371 & 0.1218 & 0.7186 & 0.4841 & $\underline{0.9592}$ & 0.1810 & 0.1608 & 0.9487 & 0.6112 & 1.2664 & & & & & \\
\hline \multirow{10}{*}{$\begin{array}{c}\text { Figure } \\
1\left(b_{1}\right)\end{array}$} & 1 & 0.0384 & 0.0441 & 0.9668 & 0.7565 & $\underline{0.9941}$ & 0.0403 & 0.0462 & 1.0129 & 0.7925 & $\underline{1.0414}$ & \multirow{10}{*}{1.7504} & \multirow{10}{*}{1.7367} & \multirow{10}{*}{0.6047} & \multirow{10}{*}{1.2794} & \multirow{10}{*}{4.8275} \\
\hline & 2 & 0.0435 & 0.0434 & 0.9667 & 0.7713 & $\underline{0.9710}$ & 0.0459 & 0.0458 & 1.0197 & 0.8136 & $\underline{1.0555}$ & & & & & \\
\hline & 3 & 0.0304 & 0.0333 & 0.9856 & 0.7942 & $\underline{1.0050}$ & 0.0308 & 0.0338 & 0.9986 & 0.8046 & $\underline{1.0183}$ & & & & & \\
\hline & 4 & 0.0384 & 0.0426 & 0.9695 & 0.7663 & $\underline{1.0173}$ & 0.0403 & 0.0446 & 1.0156 & 0.8028 & $\underline{1.0658}$ & & & & & \\
\hline & 5 & 0.1187 & 0.1194 & 0.8170 & 0.8521 & $\underline{1.0954}$ & 0.1604 & 0.1613 & 1.1036 & 1.1511 & $\underline{1.4798}$ & & & & & \\
\hline & 6 & 0.1247 & 0.1223 & 0.8764 & 0.8570 & $\underline{4.8597}$ & 0.1524 & 0.1495 & 1.0712 & 1.0474 & 5.9397 & & & & & \\
\hline & 7 & 0.1448 & 0.1509 & 0.7492 & 0.8380 & $\underline{1.1334}$ & 0.2276 & 0.2371 & 1.1774 & 1.3169 & $\underline{1.7811}$ & & & & & \\
\hline & 8 & 0.1608 & 0.1636 & 0.7365 & 0.8382 & $\underline{1.3925}$ & 0.2635 & 0.2681 & 1.2066 & 1.3732 & 2.2813 & & & & & \\
\hline & 9 & 0.1721 & 0.1709 & 0.7176 & 0.8408 & $\underline{1.3596}$ & 0.2978 & 0.2957 & 1.2418 & 1.4549 & 2.3526 & & & & & \\
\hline & 10 & 0.1714 & 0.1796 & 0.7050 & 0.7965 & 1.1200 & 0.3034 & 0.3180 & 1.2480 & 1.4099 & 1.9825 & & & & & \\
\hline
\end{tabular}

over, the false alarm rate of eigentargets is much smaller than that of FKT, when the detection rate approached $100 \%$, so the ROC curves indicate that eigentargets have a better performance than FKT.

To present further validation of the performance of eigentargets, we compare eigentargets with other detection methods such as FKT, max-median (MMed), max-mean (MMean), ${ }^{6}$ and two-dimensional LMS (TDLMS) ${ }^{7}$ by numerical values. Three metrics for small target detection, signal-to-noise ratio (SNR), signal-to-noise gain (SNRG), and background suppression factor $(B S F)$, are used to evaluate the detection performance. They are defined by: $S N R=S / N, S N R G=S N R_{\text {out }} / S N R_{\text {in }}$, and $B S F=C_{\text {in }} / C_{\text {out }} . S$ is the maximum amplitude of target signal (intensity), and $N$ is the maximum amplitude of background signal. $C_{i n}$ is the standard deviation of the original background, and $C_{\text {out }}$ is that of the target map background. Obviously, the larger the three metrics, the better the performance of the detection method is. Table 1 lists the values of three metrics for detecting the targets of Figs. $1\left(a_{1}\right)$ and $1\left(b_{1}\right)$. From Table 1, we can see that the eigentargets (ET) proposed in this letter have larger $S N R$ and $B S F$ than other methods for every target, which means thateigentargets has the best detection performance.

In conclusion, the experiments executed in this section validate that the eigentargets method can be used successfully to detect IR point targets.

\section{Conclusions}

Referring to the concept of eigenfaces, we propose a novel detection method for IR point targets based on eigentargets. The Gaussian intensity function is used to generate the training images. Moreover, we design a target map function that can normalize the Euclidian distance but not directly use the Euclidian distance. In comparison with other detection methods, the eigentargets method outperforms other detection methods with larger SNR and BSF.

\section{References}

1. A. Mahalanobis, R. Muise, S. Stanfill, and A. Nevel, "Design and application of quadratic correlation filters for target detection," IEEE Trans. Aerosp. Electron. Syst. 40(3), 837-850 (2004).

2. L. A. Chan, S. Z. Der, and N. M. Nasrabadi, "Neural based target detectors for multi-band infrared imagery," in Image Recognition and Classification, Algorithms, Systems, and Applications, B. Javidi Ed., pp. 1-36, Marcel Dekker, New York (2002).

3. M. Turk and A. Pentland, "Eigenfaces for recognition," J. Cogn Neurosci. 3(1), 71-86 (1991).

4. Zh. Liu, Ch. Chen, X. Sh, and X. Zou, "Detection of small objects in image data based on the nonlinear principal component analysis neural network," Opt. Eng. 44(9), 093604 (2005).

5. S. Sims and A. Mahalanobis, "Performance evaluation of quadratic correlation filters for target detection and discrimination in infrared imagery," Opt. Eng. 43(8), 1705-1711 (2004).

6. S. D. Deshpande, M H. Er, R. Venkateswarlu, and P. Chan, "Maxmean and max-median filters for detection of small targets," Proc. SPIE 3809, 74 (1999).

7. T. Soni, J. R. Zeidler, and W. H. Ku, "Performance evaluation of 2-D adaptive prediction filters for detection of small objects in image data," IEEE Trans. Image Process. 2(3), 327-340 (1993). 